\title{
Mercados hospitalares em área urbana: uma abordagem metodológica
}

\author{
Urban hospital markets: \\ a methodological approach
}

Rejane Sobrino Pinheiro 1,2

Cláudia Travassos 3

Dani Gamerman 4

Marília Sá Carvalho 5

\footnotetext{
1 Núcleo de Estudos de Saúde Coletiva, Centro de Ciências da Saúde, Universidade Federal do Rio de Janeiro. Av. Brigadeiro Trompowski $s / n, 50$ andar, Ala Sul, Rio de Janeiro, $R J$ 21944-590, Brasil. rejanesp@acd.ufrj.br 2 Departamento de Medicina Preventiva, Faculdade de Medicina, Universidade Federal do Rio de Janeiro. C. P. 68037, Rio de Janeiro, $R J$ 21944-970, Brasil. 3 Departamento de Informação em Saúde, Centro de Informação Científica e Tecnológica, Fundação Oswaldo Cruz, Av. Brasil 4365, sala 207b, Rio de Janeiro, $R J$ 21045-900, Brasil. claudia@malaria.procc. fiocruz.br

4 Instituto de Matemática, Universidade Federal

do Rio de Janeiro. Cidade Universitária, Centro de Tecnologia, Bloco $C$, sala 101, Rio de Janeiro, RJ 21945-970, Brasil. dani@dme.ufrj.br 5 Departamento de Epidemiologia e Métodos Quantitativos em Saúde, Escola Nacional de Saúde Pública, Fundação Oswaldo Cruz. Rua Leopoldo Bulhões 1480, 80 andar, Rio de Janeiro, RJ 21041-210, Brasil. marilia@malaria.procc. fiocruz.br
}

\begin{abstract}
This study developed a method for the construction of hospital markets in a metropolitan area, focusing on users of the Unified Health System (SUS) with hip fractures and admitted to municipal hospitals in Rio de Janeiro in 1994-1995. The study used a spatial smoothing technique based on a Kernel (quartic) estimate for constructing areas of care for each hospital and subsequently for hospital markets. Areas of the city were presented where there was a market domain and a secondary domain for treating patients with hip fractures. Hospital market analysis can help health planners organize resources in the health care system.
\end{abstract}

Key words Spatial Analysis; Health Service; Health Care Facilities; Manpower and Services

Resumo Neste trabalho, desenvolveu-se um método de construção de mercados hospitalares em região urbana, considerando os pacientes que sofreram fratura de colo do fêmur e que foram internados durante o período de 1994-1995 em hospitais do município do Rio de Janeiro financiados pelo Sistema Único de Saúde (SUS). Utilizou-se técnica de alisamento espacial, a partir da estimativa de Kernel (quártico) para construção de áreas de atendimento de cada hospital e, posteriormente, dos mercados hospitalares. Foram apresentadas áreas do município nas quais houve domínio do mercado e domínio secundário no atendimento de pacientes com fratura de colo do fêmur. O estudo de mercados hospitalares pode auxiliar o planejador de saúde na busca de realocações mais eficientes durante o processo de alocação de recursos.

Palavras-chave Análise Espacial; Serviços de Saúde; Instituições de Saúde; Recursos Humanos e Serviços 


\section{Introdução}

Uma das dimensões da qualidade de um sistema de saúde está associada ao equilíbrio entre as necessidades de cuidados de saúde da população e a oferta de serviços: desníveis entre necessidade e oferta podem gerar uso desnecessário ou demanda reprimida. Desse modo, é possível que as taxas elevadas de utilização de serviços de saúde estejam indicando a maior necessidade ou a utilização desnecessária, da mesma forma que as taxas reduzidas estejam apontando dificuldade de acesso, insuficiência de recursos ou menor necessidade de cuidados de saúde.

Apesar de a necessidade ser o fator mais importante na compreensão do uso de serviços de saúde, outros aspectos atuam nesta relação. São as diferenças no perfil de necessidades que explicam o fato de as mulheres usarem mais serviços de saúde que os homens, bem como os indivíduos nos extremos da cadeia etária. No entanto, não somente fatores biológicos estão associados ao uso, mas a condição social dos indivíduos também é fator importante na inteligibilidade de variações nas taxas de utilização dos serviços de saúde. Uma vez controlados os efeitos da demanda, as diferenças no uso dos recursos de saúde podem ser justificadas também pelas características da oferta, formas de organização dos serviços e sua modalidade de pagamento, tal como pela especialização dos profissionais, preferência dos profissionais por certos tratamentos médicos e pelos métodos diagnósticos.

As variações entre taxas de utilização de serviços de saúde têm sido observadas entre países, regiões de um mesmo país, serviços e mesmo entre profissionais. Seus motivos não estão claros e alguns autores apontam fatores relacionados à oferta (maior disponibilidade de recursos e incerteza médica), enquanto outros enfocam aqueles relacionados à demanda, como, por exemplo, a condição social.

Os estudos acerca da variação na utilização de serviços de saúde têm sido realizados com base na comparação de grandes regiões político-administrativas, tais como distritos, estados ou países. Várias destas pesquisas mostraram diferenças geográficas quanto a esse uso. Em estudo pioneiro, Wennberg \& Gittelsohn (1973) utilizaram unidades geográficas de análise construídas de forma a refletir a influência de determinado serviço hospitalar, ao qual denominaram mercado hospitalar. Mercados hospitalares são pequenas áreas definidas com base no agrupamento de unidades de área menores (códigos de endereçamento postal, quadras ou municípios), visando à criação de regiões em que a maioria da população residente utiliza certo estabelecimento de saúde. A identificação dos mercados de cada serviço, ou do conjunto de serviços, traz consigo a determinação da população que potencialmente recorre a esse serviço, permitindo o cálculo de taxas do uso de serviços de saúde. Com a abordagem adotada pelos autores, as taxas de uso de serviços de saúde refletiam as taxas de utilização da população residente na área, bem como sua relação com os recursos em saúde disponíveis e o estilo da prática dos profissionais do serviço. As variações observadas nas taxas de utilização calculadas a partir da definição de mercado hospitalar -, foram mais acentuadas do que aquelas obtidas por áreas definidas de acordo com critério político-administrativo. Para o estudo de variações no uso de serviços de saúde, considera-se inadequada a análise baseada em áreas delimitadas por critério político-administrativo, em razão de a população poder usar serviços de outras regiões.

Wennberg \& Gittelsohn (1973), em seus primeiros estudos de mercados hospitalares, agruparam cidades de onde provinham os usuários de determinados hospitais. Trabalhos mais recentes vêm agrupando unidades de áreas menores, como regiões de código de endereçamento postal (McMahon Jr. et al., 1993). Na prática, os mercados são criados a partir da unidade de área de localização do estabelecimento de saúde, agregando-se outras unidades de área em que a maioria da população residente tenha utilizado aquele estabelecimento e não outro. Por sua vez, alguns autores constróem o mercado hospitalar fundamentando-se na agregação de unidades de área das quais, pelo menos, 3\% dos pacientes do estabelecimento de saúde provenham delas (Morrisey, 1993). Essencialmente, os mercados devem conter áreas nas quais poucos moradores busquem cuidados em serviços fora daquele mercado, ao passo que poucos moradores de outras áreas procurem os serviços nele contidos. Vale ressaltar que os mercados hospitalares geralmente diferenciam-se segundo o diagnóstico e o procedimento diagnóstico ou terapêutico enfocado.

Em alguns dos trabalhos relativos a mercados hospitalares, estudaram-se áreas rurais, em que há população esparsa e, normalmente, a oferta de serviços hospitalares é hierarquizada e regionalizada (Väänänen et al., 1992; Wennberg \& Gittelsohn, 1973). Nesses exemplos, as unidades maiores - cidades ou distritos - mostravam-se como soluções viáveis para a configuração dos mercados por haver maior facilidade de relacionar a população aos estabelecimentos de saúde. Para áreas urbanas, contudo, 
as dificuldade metodológicas são maiores, pois existe mais probabilidade de ocorrer sobreposição de mercados em razão de as barreiras geográficas serem muito menores.

No presente trabalho, propõe-se um método de construção de mercados hospitalares em regiões urbanas e apresenta-se um exemplo da aplicação desse método voltado a internações para tratamento de fratura de colo do fêmur, problema de saúde descrito na literatura como de baixa incerteza médica tanto na definição do diagnóstico como na identificação dos procedimentos terapêuticos mais efetivos. A escolha de um problema com baixa incerteza na decisão médica facilita a validação da metodologia empregada - a de construção de mercados hospitalares -, pois deve gerar baixa variação nas taxas de uso entre mercados que possam ser atribuídas a fatores da oferta. Outra vantagem no uso da fratura de colo do fêmur é a quase inexistência de problemas de acesso que influenciem as taxas de uso. Como esse é um problema agudo, exigindo atendimento médico imediato e não ocasionando diretamente a morte do paciente, são raros os casos de fratura sem atendimento hospitalar.

\section{Materiais e métodos}

Foram utilizadas as informações do Sistema de Informações Hospitalares do Sistema Único de Saúde (SIH-SUS). Selecionaram-se as internações com diagnóstico principal de fratura de colo do fêmur (CID9 820 - OMS, 1985) ocorridas no Município do Rio de Janeiro em 1994 e 1995. Esse sistema tem por objetivo o pagamento dos serviços executados pelas unidades de saúde com financiamento público, cuja cobertura é bastante ampla, tendo sido calculado em, aproximadamente, $70 \%$ a $80 \%$ das internações realizadas no país (Carvalho, 1998).

No estudo, foram incluídos 16 hospitais do Município do Rio de Janeiro conveniados com o SUS, os quais são responsáveis por 2.169 internações para tratamento de fratura de colo do fêmur no período 1994-1995. Foram excluídas do estudo: as internações classificadas no procedimento como "Fora de Possibilidade Terapêutica” (FPT); as internações de pacientes residentes fora do Município do Rio de Janeiro; oito hospitais públicos que efetuaram menos de 20 internações no período estudado; além dos hospitais exclusivos de população carcerária (Departamento Geral do Sistema Penitenciário do Estado do Rio de Janeiro - DESIPE) e de profissionais do Corpo de Bombeiros e Polícia Militar.
Grande parte das internações apresentava endereço incompleto ou incorreto, o que dificultou o recurso a métodos automáticos de localização dos endereços, que foi feita manualmente. Foram localizados os endereços de $72,1 \%$ dos pacientes internados, dos quais $12,6 \%$ correspondiam a pacientes residentes em outros municípios. As internações foram situadas espacialmente nos centróides dos setores censitários.

\section{Método de construção dos mercados hospitalares}

Criou-se um mapa de pontos que representava as internações efetuadas por cada hospital no período em foco, bem como foram construídas áreas de atendimento contínuas (áreas de atuação de cada hospital) a partir de cada mapa de pontos. Utilizou-se um método de alisamento espacial - Kernel quártico - para a estimativa da área contínua de atendimento de hospitais com base em um conjunto de pontos espacialmente distribuídos (Bailey, 1995). Este método permite estimar a "intensidade de internações" em toda a superfície analisada, como função das internações de pacientes que residem próximo a esse local. Esta estimativa leva em consideração a quantidade de internações que estão ao redor, atribui maior peso às mais próximas e reduzida importância às mais afastadas, além de desconsiderar pontos fora da região de alisamento (largura de banda). Nos locais próximos ao contorno do município, as estimativas são subestimadas, uma vez que a região de alisamento o ultrapassa, incluindo artificialmente maior quantidade de locais sem internação (efeito de borda).

Foi utilizada uma largura de banda de $4.000 \mathrm{~m}$ e grade regular composta por $400 \times 200$ células. Cada célula corresponde a um local $s$, que receberá valor correspondente a cada estimativa feita, transformando o mapa de pontos em área contínua. Cada célula da grade possui $167 \mathrm{~m} \times 167 \mathrm{~m}$.

$\mathrm{O}$ alisamento espacial foi realizado com o auxílio do módulo espacial do pacote estatístico S-Plus 3.3 (Mathsoft Incorporation, 1995), não tendo sido corrigido o efeito de borda.

A intensidade de internações obtidas com o alisamento espacial aponta as áreas onde o hospital concentra o seu atendimento. Porém, as áreas de atendimento de cada hospital não levam em conta a quantidade de atendimentos em relação ao total de fraturas de colo do fêmur que teve lugar em dada região. Uma vez obtidas as áreas de atendimento de cada hos- 
pital, pode-se construir a estimativa do mercado hospitalar a partir da razão entre a intensidade de internações levadas a efeito em um hospital provenientes de determinado local $s$ e a intensidade de fraturas ocorridas nesse local. Para tal processamento, calculou-se a razão do Kernel das internações de cada hospital e do Kernel das internações totais, $\rho_{\tau}(\mathrm{s})$ (Bailey, 1995). O valor de $\rho_{\tau}$ (s) é estimativa da intensidade de internações por unidade de população fraturada, ou seja, aproximação do percentual das internações em dada unidade de área $s$ da grade que foram efetuadas por determinado hospital.

\section{Divisão dos mercados hospitalares}

Em áreas urbanas é provável que exista divisão de mercado entre hospitais, isto é, áreas nas quais a população utiliza mais de um serviço. Para discriminar quais áreas são atendidas prioritariamente por um hospital, ou se mais de um hospital dividiu a responsabilidade pelo atendimento da população de certos locais, elaborou-se um procedimento para mostrar as regiões do Município do Rio de Janeiro onde houve ou não divisão de mercado. Arbitrou-se que determinado hospital possui domínio do mercado em uma região quando interna, por exemplo, $80 \%$ dos fraturados dessa região. Já nos casos em que dois ou mais hospitais dividem a responsabilidade do atendimento (por exemplo, três hospitais cada um com densidade de atendimentos de 30\% em mesma área), considerou-se que há divisão do mercado entre os três hospitais nessa área.

Para conhecer-se, então, a divisão de mercado, calculou-se o valor máximo da razão de Kernel para cada unidade de área $s$, de todos os hospitais, obtendo-se um mapa resultante, no qual cada célula $s$ apresenta o maior valor encontrado para $\rho_{\tau}(\mathrm{s})$ dentre as células $s$ de todos os mercados hospitalares.

O mapa resultante representa a sobreposição dos mercados de cada hospital, podendose evidenciar regiões onde existiu domínio do atendimento por um hospital, regiões em que houve divisão dos atendimentos entre poucos hospitais e regiões nas quais vários hospitais foram responsáveis pelas internações ocorridas. A partir destas informações, criaram-se ainda, mapas para mercado dominante e mercado secundário, identificando os hospitais que dominaram o atendimento das fraturas de colo do fêmur locais e que apresentaram domínio secundário do mercado. Para isso, foram arbitrados pontos de corte para as estimativas das proporções de internações. Unidades de área $s$ cujos hospitais atuantes mostraram-se responsáveis por até $20 \%$ do atendimento, foram designadas como áreas de flutuação de mercado, nas quais diversos prestadores deram atendimento à população local. Proporções de atendimento entre $20 \%$ e $50 \%$ foram consideradas como relativas a mercados divididos (mercados secundários), onde poucos hospitais foram responsáveis pelo atendimento. Para proporções de atendimento acima de $50 \%$, considerou-se que um hospital dominou o mercado da referida área $s$ e as áreas de predominância foram mapeadas.

Unidades de área $s$ podem expressar ainda valores elevados em locais nos quais há baixa densidade populacional ou de usuários do SUS, uma vez que o denominador da razão de Kernel apresentaria valores pequenos, produzindo instabilidade e estimativas espúrias.

Foram eliminadas da análise as áreas de domínio de mercado muito pequenas, compostas por até 50 células da grade, o que corresponde aproximadamente a área de $1,4 \mathrm{~km}^{2}(50$ x 167 x $\left.167 \mathrm{~m}^{2}\right)$.

\section{Resultados}

A distribuição espacial dos 16 hospitais estudados é irregular, assim como a distribuição das internações não seguiu o mesmo padrão da distribuição populacional (Figura 1).

\section{Mercados hospitalares}

A Figura 2 mostra um exemplo de mercado hospitalar no qual são identificados os locais onde o hospital foi prestador importante. Observou-se que a maioria dos mercados hospitalares para tratamento de fratura de colo do fêmur na cidade do Rio de Janeiro possuiu forte atuação na área em torno da localização do próprio hospital. Porém, alguns hospitais não evidenciaram, de forma clara, um mercado com esse padrão, pois as áreas que demonstraram atuação mais importante estavam bem afastadas da localização do hospital. Em alguns casos, esse padrão resulta da baixa freqüência de casos, dado que o algoritmo produz valores elevados em locais onde há número muito baixo de internações no SUS ou em que existe baixa densidade populacional.

\section{- Divisão do mercado}

Observou-se que metade do município, aproximadamente, possuiu um prestador, pelo menos, com domínio do mercado, isto é, áreas nas 
Mapa do Município do Rio de Janeiro, apresentando as internações para fratura de colo de fêmur, segundo local de residência, e os 16 hospitais do Município do Rio de Janeiro, conveniados com o SUS, que realizaram estas internações, 1994 a 1995.
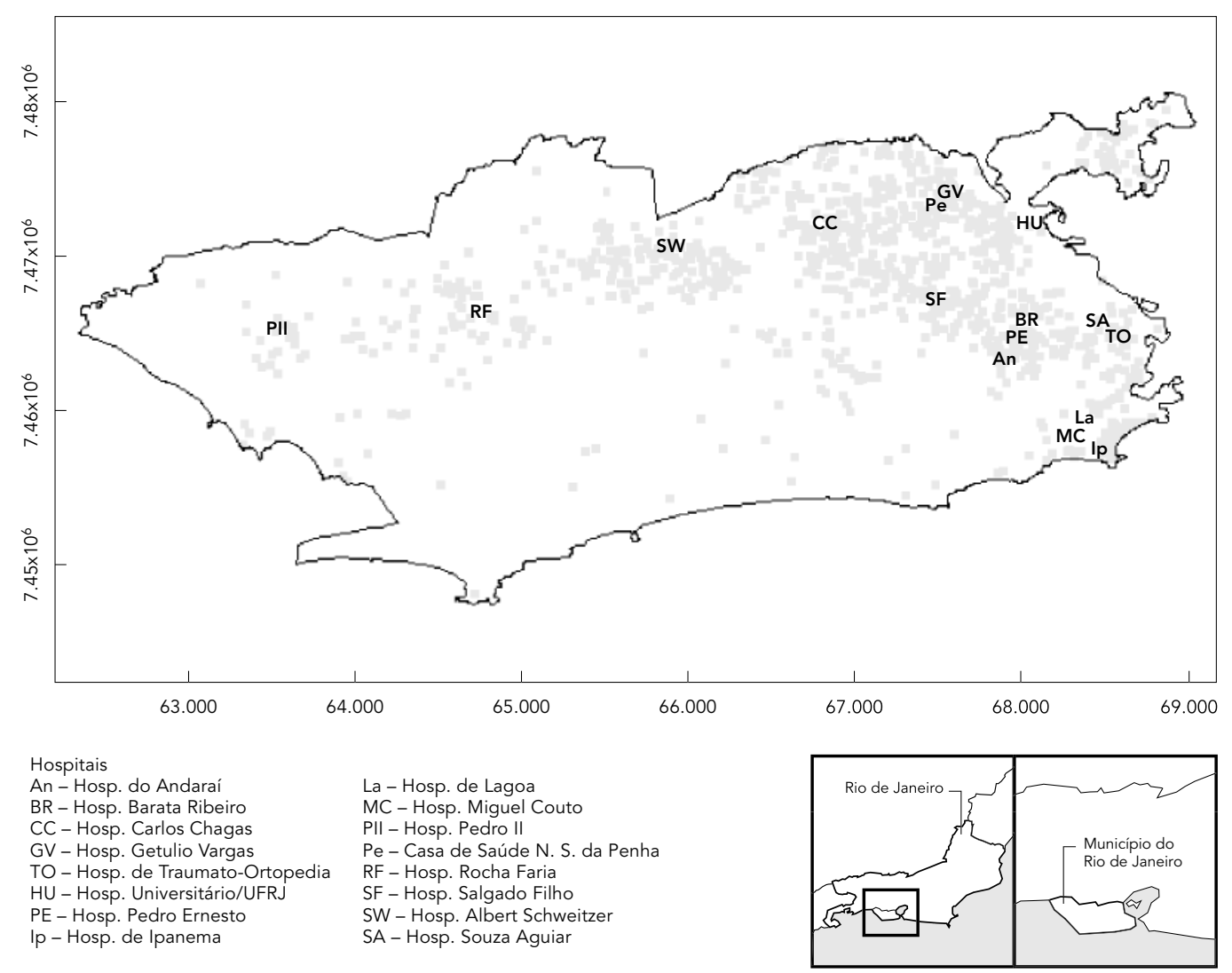

UFRJ $=$ Universidade Federal do Rio de Janeiro.

quais mais de $50 \%$ dos pacientes analisados foram atendidos em um único hospital (Figura 3).

O mapa de divisão de mercados mostrou área clara, de alta divisão de mercados (máximo de proporção de internação de todos os mercados nesta região foi de até 0,20$)$. Em volta dessa área, houve outra com máximo de 0,30, dando a conhecer ainda alto compartilhamento do atendimento entre hospitais. Observouse área extensa, que corta o município transversalmente, na qual alguns prestadores dividiram mercados e em que o hospital mais importante foi responsável por $40 \%$ a $50 \%$ dos atendimentos. As áreas mais escuras indicaram que ali existiu um prestador de forte atuação, praticamente dominando o mercado dessa região (máximo entre 0,6 e 0,8). As áreas em preto tenderam a apresentar interpretação semelhante. Porém, deve-se ter cuidado ao analisá- las, pois algumas representaram regiões onde houve pequeno número de internações, podendo estar indicando baixa densidade populacional ou baixa proporção de residentes que utilizaram o SUS em lugar de domínio do mercado por um prestador.

Na área mais clara do mapa - que corresponde às regiões da Tijuca, Maracanã, Vila Isabel, Andaraí e Grajaú, destacada em parágrafo anterior - existe grande concentração de hospitais, tendo sido aí que ocorreu a pulverização do atendimento entre diferentes prestadores. Praticamente todos os hospitais também realizaram atendimentos de pacientes residentes nessa área.

Apesar de igualmente existir concentração de hospitais na Zona Sul, a divisão dos mercados não se deu de modo equivalente ao observado nos hospitais das regiões acima, isto é, 
Mercado hospitalar para fratura de colo de fêmur do Hospital Universitário Clementino Fraga Filho (HU-UFRJ)، 1994 a 1995

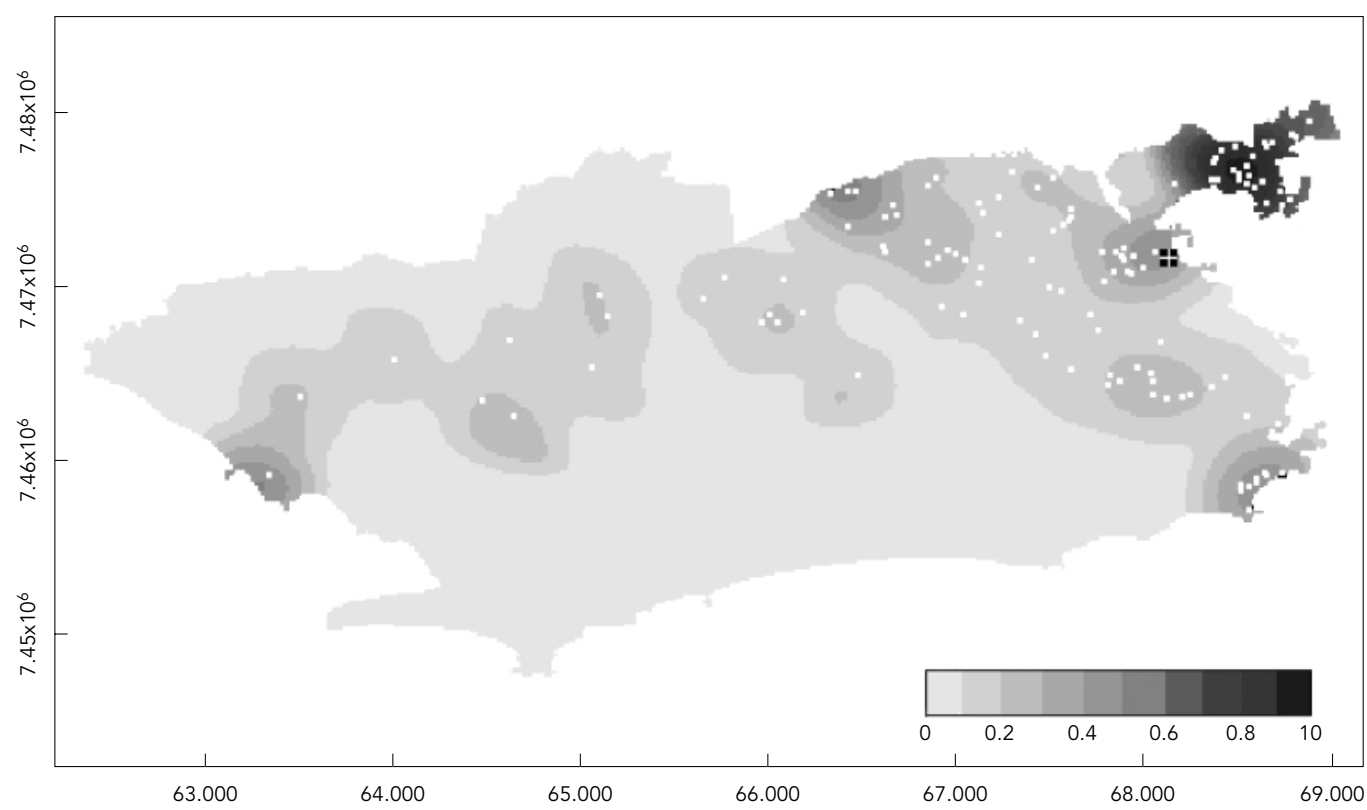

Figura 3

Divisão de mercados hospitalares para fratura de colo de fêmur, nas diversas regiões do Município do Rio de Janeiro, 1994 a 1995.

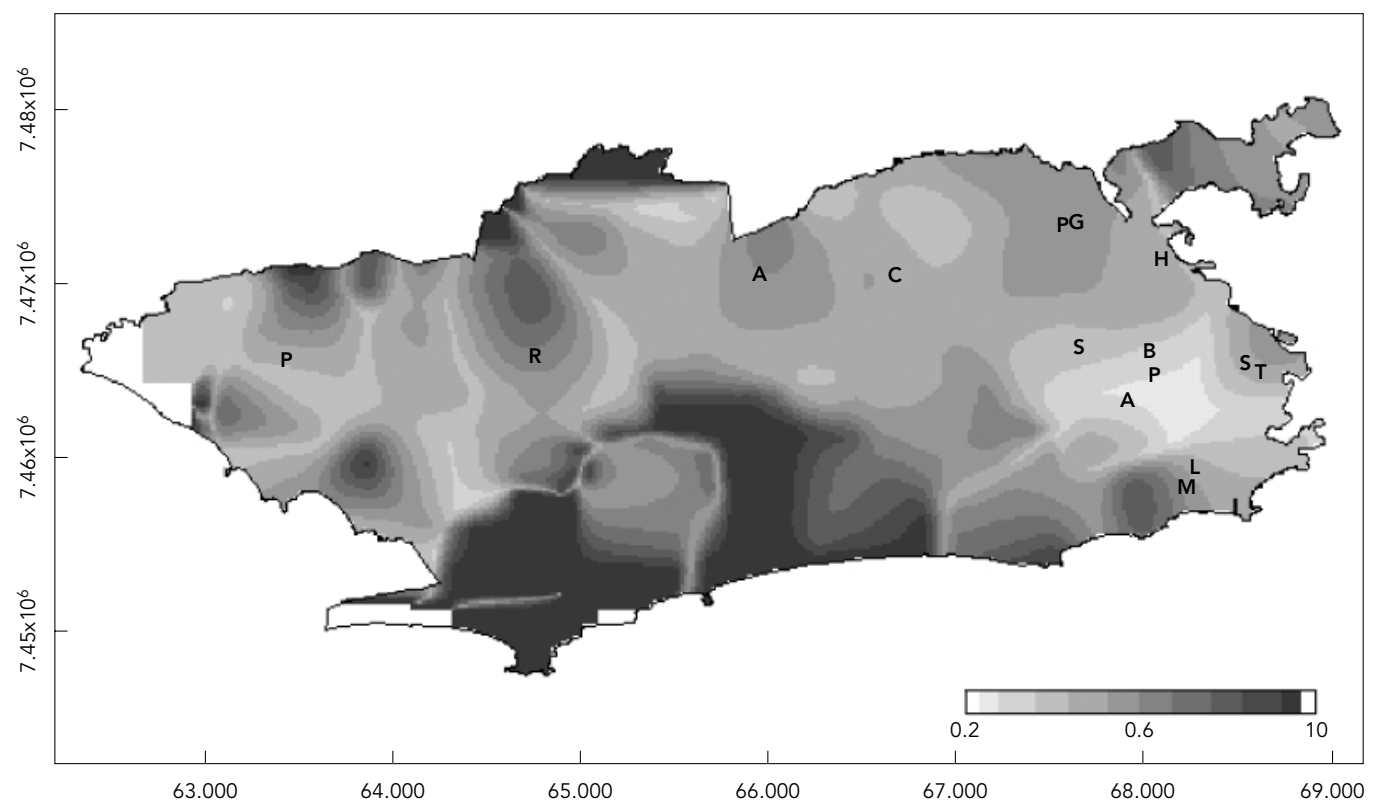


houve predomínio de um hospital na região. Apesar de os hospitais terem localização próxima um do outro, não dividiram o mercado de forma equilibrada.

\section{Hospitais com domínio do mercado}

Para identificar quais hospitais apresentaram atuação mais importante no Município do Rio de Janeiro, foi elaborado um mapa que representava as áreas de domínio de mercado, utilizando-se tonalidades diferentes para cada um dos hospitais (Figura 4). Observou-se que seis hospitais evidenciaram domínio de mercado, responsabilizando-se por mais de $50 \%$ das internações ocorridas em certas regiões.

\section{Hospitais com mercados secundários}

A área em cinza corresponde às regiões do $\mathrm{Mu}$ nicípio do Rio de Janeiro onde existiu divisão do mercado, ou seja mais de um hospital foi respon- sável pelo atendimento sem, no entanto, haver prestador que dominasse o mercado na região.

Para facilitar a visualização dos mercados dominantes e mercados secundários, desmembrou-se o mapa em três partes. A primeira mostra, separadamente, os mercados dos hospitais localizados nas Zonas Norte, Centro e Sul do município (Figura 5). O segundo, os mercados hospitalares na Zona Oeste (Figura 6). O terceiro apresenta o mercado da Casa de Saúde Nossa Senhora da Penha, prestador que dominou o atendimento das fraturas de colo do fêmur na cidade do Rio de Janeiro (Figura 7).

O mercado hospitalar do Hospital Rocha Faria ocupa a maior parte da Zona Oeste (Figura 6). Ele domina o atendimento em duas regiões e divide o atendimento basicamente com dois outros hospitais que não realizaram procedimento cirúrgico, tratamento recomendado para a correção da fratura de colo do fêmur.

Por sua vez, o mercado hospitalar da Casa de Saúde Nossa Senhora da Penha, único hos-

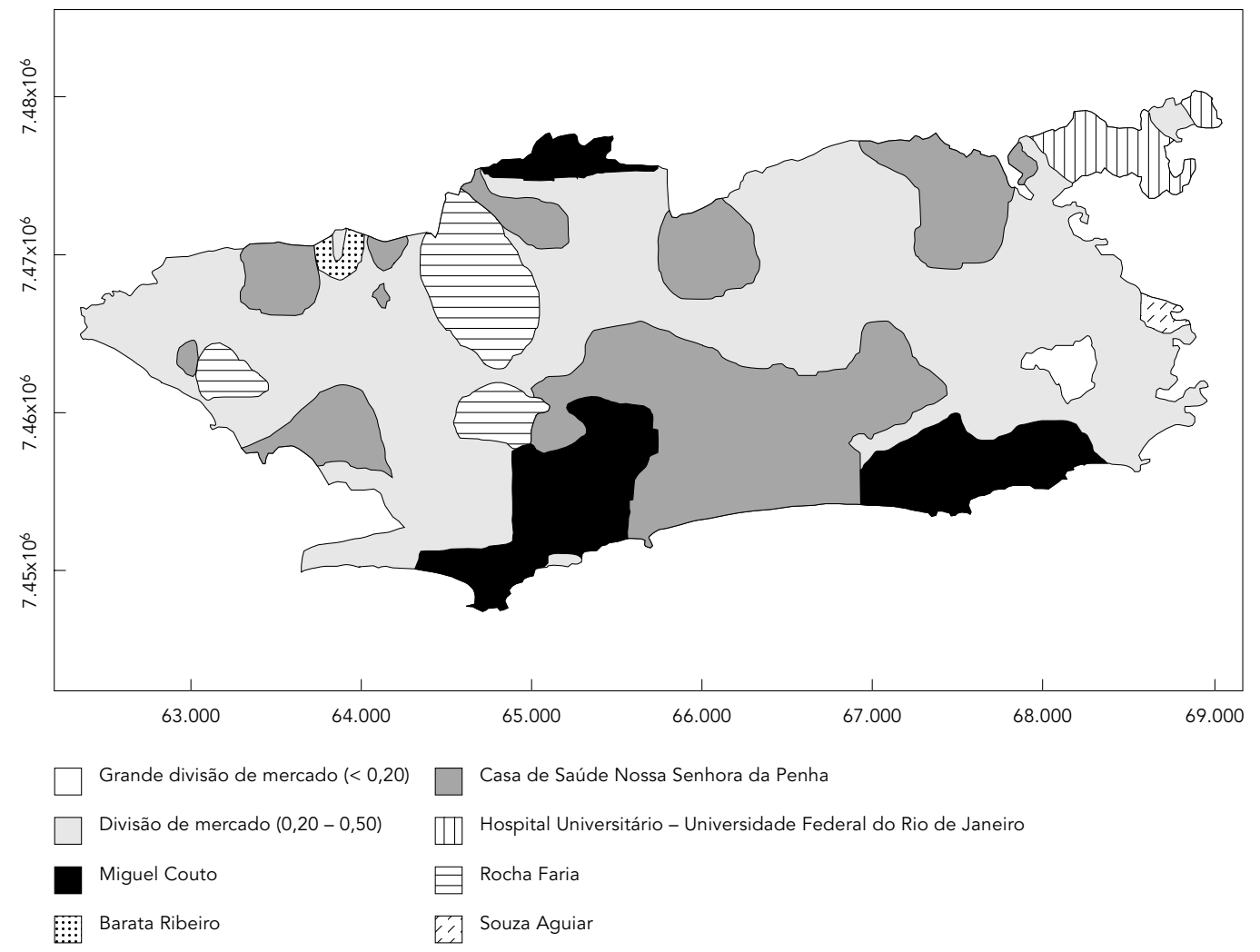




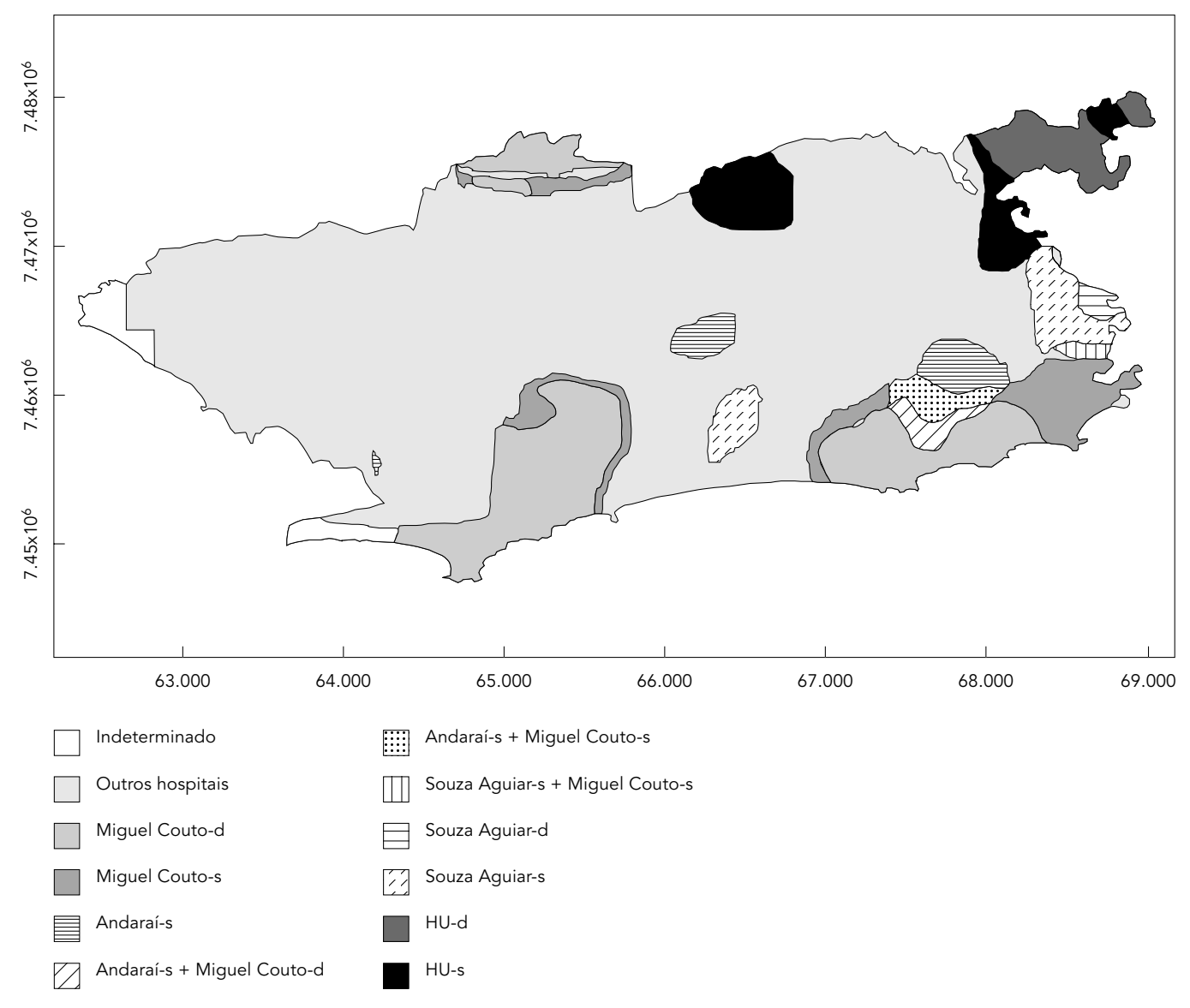

HU-UFRJ = Hospital Universitário - Universidade Federal do Rio de Janeiro. $\mathrm{d}=$ dominante; $\mathrm{s}=$ secundário.

pital privado contratado com fins lucrativos na amostra deste estudo, é o maior mercado hospitalar para tratamento de fratura de colo do fêmur na cidade do Rio de Janeiro e abrange a maior parte da extensão do município. Esse hospital efetuou cerca de $40 \%$ das internações por fratura do colo do fêmur financiadas pelo SUS na cidade. Em apenas poucas áreas, a Casa de Saúde Nossa Senhora da Penha não se destacou como prestador importante (Figura 7). Sua importância foi inexpressiva somente naquelas áreas em que havia prestadores de forte atuação (áreas em branco).

\section{Discussão}

A construção de mercados hospitalares tem por fim analisar a existência de variações nas taxas de uso de procedimentos médicos e diag- nósticos específicos e busca identificar os fatores que explicam estas variações, no intuito de corrigir eventuais excessos ou deficits no uso de serviços de saúde (Wennberg, 1985).

Em áreas urbanas, a construção de mercados hospitalares apresenta algumas dificuldades, pois a possibilidade de descontinuidades e interseções entre mercados de diferentes hospitais é bastante acentuada. A utilização da metodologia convencional (Wennberg \& Gittelsohn, 1973) é inviável na cidade do Rio de Janeiro. Neste estudo, desenvolveu-se uma metodologia para a identificação de mercados hospitalares em áreas urbanas que permitiu criar áreas contínuas de atuação dos hospitais em região urbana - mais especificamente, em uma grande metrópole - como primeiro passo para a identificação dos mercados hospitalares.

A construção de mercados hospitalares auxilia no planejamento do setor saúde, criando 


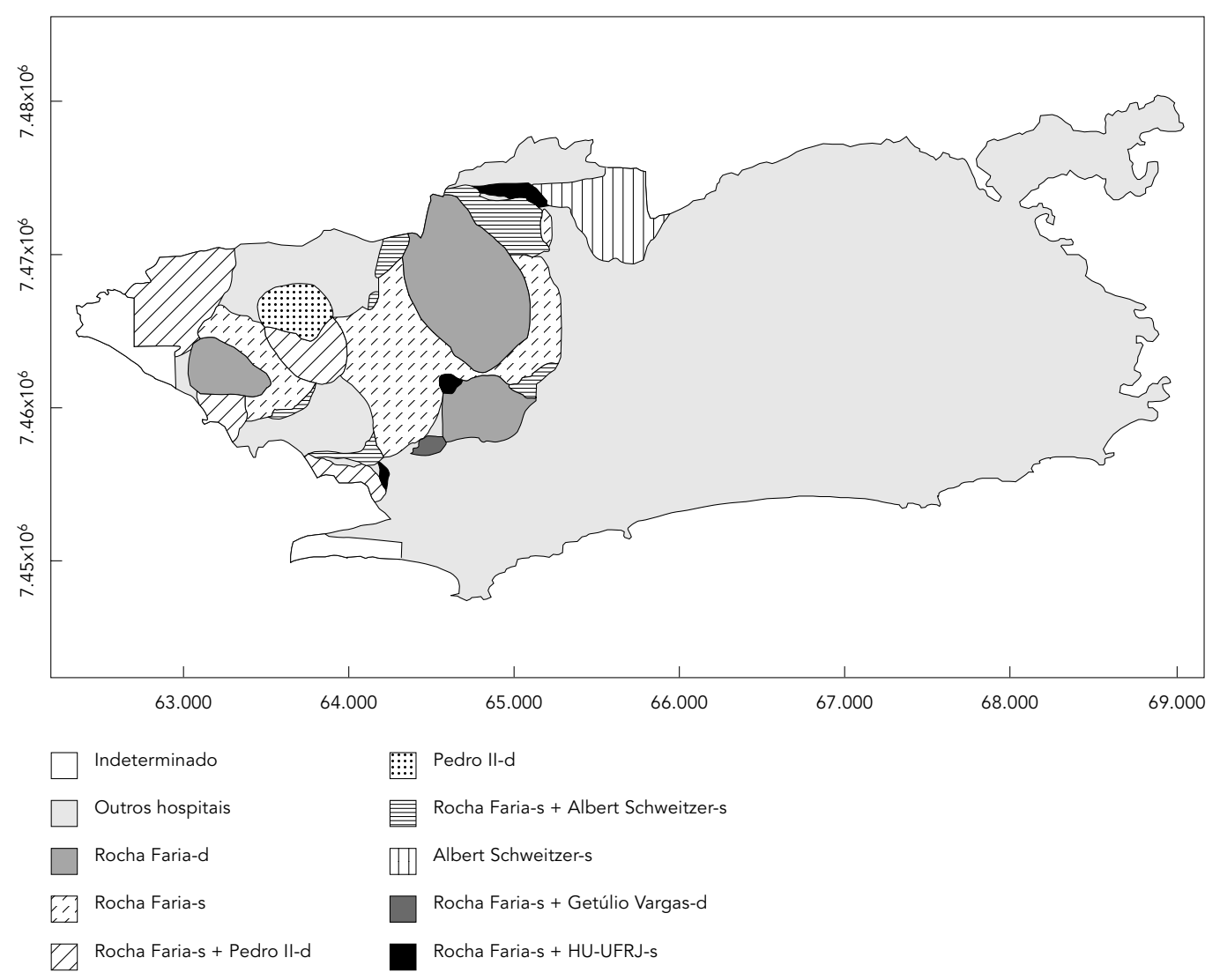

HU-UFRJ = Hospital Universitário - Universidade Federal do Rio de Janeiro. $\mathrm{d}=$ dominante; $\mathrm{s}=$ secundário.

bases para o estudo dos perfis de oferta e sua adequação à demanda, além de permitir a comparação, entre prestadores, de taxas de utilização de serviços para diagnósticos ou procedimentos médicos específicos, e estudos de avaliação dos resultados dos cuidados hospitalares em associação com as condições de saúde da população. Outra vantagem da visualização dos mercados hospitalares é a informação a respeito do fluxo de pacientes. Esta constitui uma forma alternativa de transmissão desse tipo de informação, em que o diagrama de flechas é o modo mais conhecido para representar, em mapas, os deslocamentos de pessoas entre áreas. $\mathrm{O}$ método permitiu ainda identificar que o atendimento hospitalar foi voltado, em grande parte, para pacientes residentes em áreas próximas ao hospital, mostrando a preferência dos indivíduos pelos serviços locais.

Note-se que, dos dezesseis hospitais em que houve atendimento a pacientes com fratu- ra de colo do fêmur na cidade do Rio de Janeiro, apenas seis - Miguel Couto, Barata Ribeiro, Casa de Saúde Nossa Senhora da Penha, Hospital Universitário Clementino Fraga Filho da Universidade Federal do Rio de Janeiro (HUUFRJ), Rocha Faria e Souza Aguiar - evidenciaram domínio de mercado, isto é, foram responsáveis pelo atendimento de mais de $50 \%$ das fraturas ocorridas em determinada área. Dentre estes, um único hospital - Casa de Saúde Nossa Senhora da Penha - respondeu por, aproximadamente, $40 \%$ do total de atendimentos. Este hospital apresentou domínio de mercado em várias regiões do município e mercado secundário em quase todo o restante das regiões. Sua atuação foi inexpressiva somente naquelas áreas que expuseram prestadores de forte atuação, como os hospitais Miguel Couto, Souza Aguiar, Rocha Faria e HU-UFRJ.

Além dos hospitais acima, que também exibiram áreas onde tinham domínio secundário 


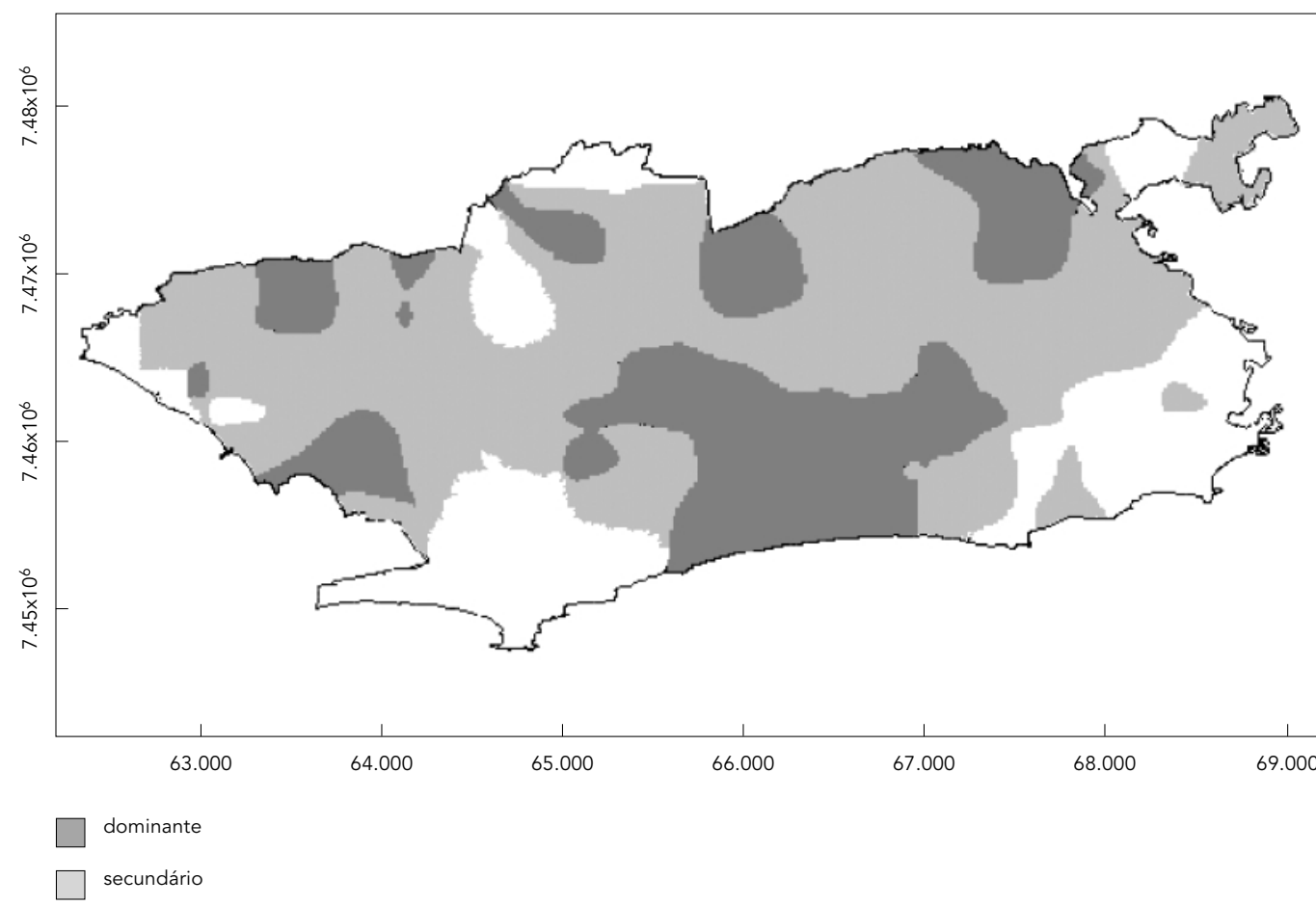

do mercado, seis outros hospitais apresentaram apenas domínio secundário de mercado Getúlio Vargas; Andaraí; Salgado Filho; Albert Schweitzer; Pedro II e Pedro Ernesto (HUPE). Já, os hospitais Ipanema, Lagoa, Carlos Chagas, e HTO (Instituto de Traumato-Ortopedia) mostraram baixa capacidade de captação de pacientes e foram responsáveis pelo atendimento de menos de $20 \%$ dos pacientes com fratura do colo do fêmur residentes nas áreas estudadas da cidade do Rio de Janeiro.

Entretanto, é preciso ressaltar que o desempenho acima pode resultar, em parte, de subnotificação de internações ao SIH-SUS. Supõese que os hospitais federais, em particular, deixem de informar a ocorrência de internação na medida em que não são remunerados pelo mesmo mecanismo de pagamento que remunera os demais hospitais do SUS.

O método proposto não eliminou o problema de instabilidade nos resultados em decorrência de baixa densidade populacional ou de fraturados em certas áreas. Em virtude de valores reduzidos nos denominadores dos cálculos dos mercados hospitalares, valores espúrios podem ser observados em função de artifício matemático, em lugar de estarem representando atuação importante de um hospital em determinada área.

Quando são eliminadas as áreas de domínio de mercado em regiões onde há baixa densidade demográfica ou baixa proporção de internações, buscando eliminar as instabilidades decorrentes de limitações do método, obtémse outro quadro na caracterização dos mercados hospitalares: os hospitais Barata Ribeiro, Andaraí, Getúlio Vargas e Pedro Ernesto parecem perder sua condição de domínio ou domínio secundário de mercado. Ou seja, passam também a responder por menos de $20 \%$ do atendimento aos pacientes fraturados residentes nas áreas analisadas.

Concluindo, este estudo de mercados hospitalares pode auxiliar o planejador de saúde na busca de realocações mais eficientes durante o processo de alocação de recursos. A pulverização do atendimento observada em algumas áreas da cidade indica que pessoas residentes em uma única área são atendidas por diversos hospitais, muitas vezes localizados em 
regiões distantes. Apesar de parte dos casos de fratura do colo do fêmur ser decorrente de acidentes que tiveram lugar fora do domicílio, supõe-se que a maioria dessas fraturas ocorre em população idosa, no próprio domicílio ou na proximidade deste. A literatura tem relatado que hospitais que executam altos volumes de determinados procedimentos médicos tendem a fazê-lo com maior qualidade do que os hospitais que executam pouca quantidade desses procedimentos (Luft et al., 1990). Uma opção mais eficiente seria centralizar o atendimento em número menor de hospitais, definindo prestadores prioritários por regiões da cidade. Esta estratégia deveria ser acompanhada da im- plantação de um sistema para o monitoramento do desempenho desses serviços.

Estudos futuros devem buscar aprimorar o método de forma a reduzir as instabilidades. Outra questão a ser tratada em trabalhos posteriores é o processamento que inclui o efeito de borda, o qual, como apontado anteriormente, produzirá mercados hospitalares pouco diferenciados dos obtidos neste trabalho, principalmente para os hospitais localizados próximos às fronteiras do município e os que internaram muitos pacientes residentes nessas fronteiras. Mercados diferentes também devem ser obtidos por intermédio do estudo de outras condições clínicas ou de procedimentos médicos.

\section{Referências}

BAILEY, T. C. \& GATRELL, A. C., 1995. Interactive Spatial Data Analysis. Essex: Longman.

CARVALHO, D. M., 1998. Sistema de Informação e Alocação de Recursos: Um Estudo sobre a Possibilidade de Uso de Grandes Bases de Dados Nacionais para Alocação Orientada de Recursos. Dissertação de Mestrado, Rio de Janeiro: Instituto de Medicina Social, Universidade do Estado do Rio de Janeiro.

LUFT, H. S.; GARNICK, D. W.; MARK, D. H. \& McPHEE, S. J., 1990. Hospital Volume, Physician Volume, and Patient Outcomes: Assessing the Evidence. Ann Arbor: Health Administration Press Perspectives.

MATHSOFT INCORPORATION, 1995. S-Plus for Windows, Version 3.3. Seattle: Mathsoft Inc.

McMAHON Jr., L. F.; WOLFE, R. A.; GRIFFTH, J. R. \& CUTHBERTSON, D., 1993. Socioeconomic influence on small area hospital utilization. Medical Care, 31(Sup. 5):YS29-YS36.

MORRISEY, M. A., 1993. On definig small areas. Medical Care, 31(Sup. 5):YS89-YS95.

OMS (Organização Mundial de Saúde), 1985. Manual da Classificação Estatística Internacional de Doenças, Lesões e Causas de Óbitos - 9ạ Conferência de Revisão. São Paulo: Centro Brasileiro de Classificação de Doenças em Português.

VÄÄNÄNEN, I. S.; HÄRÖ, A. S.; VAUHKONEN, O. \& MATTILA, A., 1992. El grado de utilización de los hospitales y la selección de pacientes en el sistema de hospitales regionales de Finlandia. In: Investigaciones sobre Servicios de Salud: Una Antología (Organización Panamericana de Salud OPS, org.), Publicación Científica 534, pp. 462479, Washington, DC: OPS.

WENNBERG, J. E. \& GITTELSOHN, A., 1973. Small area variations in health care delivery. Science, 182:1102-1108.

WENNBERG, J. E., 1985. On patient need, equity, supplier-induced demand, and the need to assess the outcome of common medical practices. Medical Care, 23:512-520. 